\title{
Potentially Harmful Medication Use and Decline in Health- Related Quality of Life among Community-Dwelling Older Adults
}

\author{
Kenya Ie $^{1,4}\left([) \cdot\right.$ Eric Chou $^{2} \cdot$ Richard D. Boyce ${ }^{2} \cdot$ Steven M. Albert $^{3}$
}

Published online: 8 November 2017

(c) The Author(s) 2017. This article is an open access publication

\begin{abstract}
Background Several scales to quantify the impact of potentially harmful medications (PHMs) have been shown to predict mortality and functional decline; however, the effect of PHMs on quality of life (QoL) has not been well-studied.

Objective The aims of this study were to investigate an association between PHM use and change in health-related QoL among community-dwelling older adults, and to compare the predictive capacity of PHM scales.

Methods We conducted a retrospective cohort study using prescription claims data and survey responses. A total of 426 community-dwelling adults aged 65 years or older who visited senior centers and had received prescriptions through a statewide prescription drug subsidy program were included. Anticholinergic Cognitive Burden (ACB), Drug Burden Index-sedative component (DBI-Se), Drug Burden Index-anticholinergic component (DBI-ACh), and the number of regular medications and Beers list medications were calculated from the claims data between
\end{abstract}

Electronic supplementary material The online version of this article (https://doi.org/10.1007/s40801-017-0123-8) contains supplementary material, which is available to authorized users.

Kenya Ie

iekenya0321@gmail.com

1 Department of Family Medicine, University of Pittsburgh, 3518 Fifth Avenue, Pittsburgh, PA 15213, USA

2 Department of Biomedical Informatics, University of Pittsburgh, 5607 Baum Blvd, Pittsburgh, PA 15206, USA

3 Department of Behavioral and Community Health Sciences, University of Pittsburgh Graduate School of Public Health, 130 De Soto Street, Pittsburgh, PA 15261, USA

4 Present Address: Kawasaki Municipal Tama Hospital/St. Marianna University School of Medicine, 1-30-37 Shukugawara, Kawasaki, Kanagawa 214-8525, Japan baseline and 12 months. In addition, change in the EuroQoL five-dimensions questionnaire (EQ-5D) between baseline and 6- and 12-month follow-up were measured as the main outcome. A linear mixed model was used for the analysis.

Results After adjusting for covariates, both DBI-Se (coefficients $-0.076,95 \%$ confidence interval $[\mathrm{CI}]-0.131$ to -0.020 ) and DBI-Ach (coefficients -0.095 , 95\% $\mathrm{CI}-0.188$ to -0.002 ) significantly predicted a decline in EQ-5D index. The ACB, number of regular medications, and number of Beers medications did not have a significant association with EQ-5D changes.

Conclusions PHM measures incorporating dose revealed a better predictive capacity for QoL change. Reducing cumulative drug dose, as well as stopping medications, would be important for the well-being of this population.

\section{Key Points}

Our study found a high prevalence of potentially harmful medication (PHM) exposure among community-dwelling older adults.

The proportion of participants with at least one PHM use was $58.7 \%$ for Anticholinergic Cognitive Burden, 36.6\% for Drug Burden Index-sedative properties, $24.1 \%$ for Drug Burden Indexanticholinergic properties, and $19.2 \%$ for the American Geriatrics Society Beers criteria.

Among the above criteria, Drug Burden Index significantly predicted a decline in quality of life among community-dwelling older adults after adjusting for other covariates. 


\section{Introduction}

Despite an increasing awareness of potentially harmful medications (PHMs), their usage has become widespread, along with a growing chronic disease epidemic among aging populations [1]. Studies conducted in outpatient settings in several countries consistently report a PHM prevalence of $30 \%$ or higher, using different PHM criteria [2-5]. There is some evidence that PHM burden, especially the anticholinergic [6] and sedative effects [7], is associated with adverse drug events, decline in physical function, and excessive healthcare utilization [8-10]. Based on an estimation, medication-related problems are found to cost the US more than $\$ 170$ billion every year [9].

PHMs are defined in several drug lists, such as the American Geriatrics Society Beers criteria [11] and the STOPP/START criteria [12]. In addition to these criteria, several scales have been proposed to quantify the burden of medications on older adults, especially with a focus on anticholinergic effects and sedative effects. One of the widely used scales is the Drug Burden Index (DBI) [13], which was developed as a measure of overall exposure to medications with anticholinergic (DBI-Ach) and sedative (DBI-Se) properties; the scale implements the principle of dose response to quantify the medication burden. Previous studies have shown associations between higher DBI scores and lower physical function [13-16], lower cognitive function [16], increased hospital admissions [17], and a higher fall rate [18].

The Anticholinergic Cognitive Burden (ACB) [19] is another widely accepted PHM scale that uses ordinal scores to rank medications according to their clinically relevant cognitive effects. Based on a community-based observational study, every 1-point increase in the ACB score was associated with a decline in the Mini-Mental State Examination (MMSE) of 0.33 points over 2 years and a $26 \%$ increase in the risk of death [20]. ACB scores have also been shown to predict functional impairment and low cognitive performance [21, 22]. In addition to the DBI and ACB, polypharmacy (i.e. the concomitant use of more than a certain number of drugs) is a simple, albeit potentially effective, predictor of PHM use [23-27]. Furthermore, polypharmacy itself has been shown to predict excess deaths [28], increased fall risk [29], and increased readmission rates [30].

As stated above, PHM scales have been shown to predict healthcare outcomes such as functional decline [13-16], hospital admission [17], and mortality [20, 28]; however, the effect of PHMs on quality of life (QoL) has not been well-studied. This is surprising considering that PHMs may lead to adverse drug events that affect patients' morbidity, which in turn greatly influences QoL, and that QoL is a fundamental aspect of patient-centered care. Bosboom et al. found that DBI scores $>0$ and the number of medications $>5$ both predicted a decline in QoL among nursing facility residents with a diagnosis of dementia [31]. On the contrary, Franic and Jiang reported that the use of Beers list PHMs was not a significant predictor of QoL among community-dwelling older adults [32]. To our knowledge, no study has examined the association between quantified PHM scores and change in QoL among communitydwelling older adults. Given the prevalence of PHM use among older adults, it is vital to understand its impact on QoL within a community context.

The objectives of this study were to investigate associations between PHM use and change in health-related QoL among community-dwelling older adults and to compare the predictive capacity of several PHM scales.

\section{Methods}

\subsection{Study Design and Setting}

We conducted a retrospective cohort study using data collected for Falls Free PA, an interventional study comparing falls incidence between participants of Pennsylvania's Healthy Steps for Older Adults (HSOA) program and a control group of older adults who did not participate in HSOA but visited the same senior centers. For our current study, the Falls Free PA data were linked to individuallevel prescription claims data from Pennsylvania's Pharmaceutical Assistance Contract for the Elderly (PACE) program. The original Falls Free PA was conducted from 2010 to 2013 and the University of Pittsburgh Institutional Review Board approved the protocol. The protocol details and results have been published elsewhere [33].

\subsection{Study Sample}

Falls Free PA included a total of 1829 patients aged 50 years or older who visited senior centers and participated in Pennsylvania Department of Aging programs. Potential participants who were unable to provide informed consent, spoke languages other than English or Spanish, and were unable to participate in telephone follow-up calls were excluded [33]. Among the Falls Free PA participants, those who also participated and utilized Pennsylvania's PACE program between September 2010 and March 2012 were included in our analysis. Eligibility criteria for Pennsylvania's PACE program were (1) adults aged 65 years or older; (2) Pennsylvania residency for at least 90 days; (3) not being enrolled in the Department of Human Service's Medicaid prescription benefit, (4) and a 
total income of $\$ 14,500$ or less (for a married couple, combined total income must be $\$ 17,700$ or less) [34]. As of January 2015, 106,548 older adults were enrolled in the PACE program in Pennsylvania [35].

\subsection{Outcome Measurements}

The primary outcome measurement of interest was the EuroQoL five dimensions three-level version (EQ-5D-3L) summary index [36] at baseline and 6- and 12-month follow-up. The EQ-5D-3L is a standardized measurement for generic health-related QoL that has five subdomain scores consisting of mobility, self-care, usual activities, pain/discomfort, and anxiety/depression. Each of the five domains assesses one of three responses based on levels of severity (no problems/some or moderate problems/extreme problems). EQ-5D-3L health state responses can be summarized into a single summary index based on response profiles, ranging from 0 (death) to 1 (complete health), by applying weighted scores for each dimension. The EQ-5D$3 \mathrm{~L}$ has been validated in diverse patient populations in multiple settings [37].

\subsection{Exposure Variables: Potentially Harmful Medication Use}

The following five PHM scales were calculated based on the PACE prescription claims data between baseline and the 12-month follow-up: cumulative $\mathrm{ACB}$, cumulative DBI-Se, cumulative DBI-ACh, number of regular medications, and number of PHMs listed on the 2015 American Geriatrics Society 2015 updated Beers criteria [11]. For the PHM scale calculation, we included all forms of oral, suppository, and transdermal medications.

First, we calculated the three cumulative indices (ACB, DBI-Se, and DBI-ACh), which utilize duration of drug exposure. The duration of exposure to each individual prescription drug were summed during the study period and then divided by days between baseline and the 12-month follow-up, adapting an equation previously described by Salahudeen et al. [38]. The cumulative ACB was calculated based on the following equation:

$\mathrm{ACB}_{\mathrm{i}}=\frac{\sum_{i a=i 1}^{i k}(\mathrm{ACB} \text { weight for drug a } \times \text { Days Supply })}{\text { Days between Baseline and } 12 \text { month }}$

where ' $i$ ' is the subject, and ' $a$ ' is the drug name listed in the ACB list (see electronic supplementary material [ESM] $1)$.

In addition to the time of exposure, the cumulative DBISe and DBI-ACh also considers cumulative drug dose. Based on the original DBI equation described by Hilmer et al. [13] and a modified version by Salahudeen et al. [38], we calculated the DBI-Se and DBI-ACh using the following equations. Medications that were prescribed on a short-term, as needed basis were excluded from the DBI calculation:

DBI_Se $e_{i}=\frac{\sum_{i s=i 0}^{i k}\left(\frac{D_{\mathrm{s}}}{\delta_{\mathrm{s}}+D_{\mathrm{s}}} \times \text { Days Supply s }\right)}{\text { Days between Baseline and } 12 \text { month }}$

where ' $\mathrm{s}$ ' is the drug name listed in the DBI-Se list, ' $D$ ' is the daily dose of the drug, and $\delta$ is the minimum effective dose of the drug (see ESM 2),

DBI_Ach $_{i}=\frac{\sum_{i b=i 1}^{i k}\left(\frac{D_{\mathrm{b}}}{\delta_{\mathrm{b}}+D_{\mathrm{b}}} \times \text { Days Supply b }\right)}{\text { Days between Baseline and } 12 \text { month }}$

where ' $\mathrm{b}$ ' is the drug name listed in the DBI-ACh list (see ESM 3).

All the cumulative indices (ACB, DBI-Se, and DBI$\mathrm{ACh}$ ) used in this study were calculated based on the above equations.

Second, we identified the number of distinct regular medications and regular PHMs listed on the Beers list. For the purposes of the current study, we considered prescription drugs for which claims were provided for at least 30 days and at least twice during the study period to be regular drugs. Study participants were also asked to report the number of regular drugs at the 6-month follow-up for verification purposes. For our measurement, we adopted the list of drugs that were recommended to be avoided in older adults as per Table 2 of the American Geriatrics Society 2015 updated Beers criteria [11] (ESM 4). Due to data limitations, we did not include drugs, with exceptions, that require laboratory results or prescription indications (e.g. doxazosin; avoid use as an antihypertensive).

\subsection{Other Study Measures}

We collected baseline patient characteristics, including age, sex, ethnicity/race, marital status, living situation, education, income level, use of assistive devices for walking, recent history of falls, and self-reported comorbidity data. We also calculated the baseline number of comorbidities based on dichotomized responses with regard to the following 17 conditions: stroke, diabetes mellitus, hypertension, heart attack, macular degeneration, arthritis, osteoporosis, glaucoma, inner ear problem, depression, chronic obstructive pulmonary disorder or asthma, congestive heart failure, peripheral neuropathy, Parkinson's disease, cognitive impairment, any fracture after 50 years of age, and a current or previous history of cancer. In addition, participants' baseline memory impairment test scores were recorded as a covariate. 
Table 1 Baseline characteristics of study participants

\begin{tabular}{|c|c|}
\hline & PACE participants $[N=426]$ \\
\hline Age, years [mean $(\mathrm{SD})]$ & $78.57(6.72)$ \\
\hline Male sex & $48(11.27)$ \\
\hline Hispanic/Latin & $5(1.17)$ \\
\hline \multicolumn{2}{|l|}{ Race $^{\mathrm{a}}$} \\
\hline American Indian/Alaskan Native & $1(0.23)$ \\
\hline Asian & $1(0.23)$ \\
\hline Black & $33(7.75)$ \\
\hline White & $388(91.08)$ \\
\hline Other & $2(0.47)$ \\
\hline \multicolumn{2}{|l|}{ Marital status ${ }^{\mathrm{b}}$} \\
\hline Single & $44(10.33)$ \\
\hline Married & $44(10.33)$ \\
\hline Separated & $2(0.47)$ \\
\hline Widowed & $302(70.89)$ \\
\hline Divorced & $32(7.51)$ \\
\hline Live with someone & $108(25.35)$ \\
\hline \multicolumn{2}{|l|}{ Education } \\
\hline Never attended school & - \\
\hline Grade $1-8$ & $18(4.23)$ \\
\hline Grade $9-11$ & $68(15.96)$ \\
\hline Grade 12 or GED & $244(57.28)$ \\
\hline College 1-3 & $74(17.37)$ \\
\hline College 4 or more & $22(5.16)$ \\
\hline Income-sufficient for daily living ${ }^{c}$ & $293(68.78)$ \\
\hline Living in an urban area & $279(65.49)$ \\
\hline Fall in the last year & $135(31.69)$ \\
\hline Fall in the past 30 days & $34(7.98)$ \\
\hline Use of assistive devices & $136(31.92)$ \\
\hline Self-rated mobility [mean (SD) $]^{\mathrm{d}}$ & $2.77(1.03)$ \\
\hline Self-rated balance $[\text { mean }(\mathrm{SD})]^{\mathrm{d}}$ & $3.01(0.96)$ \\
\hline Number of comorbidities (per total) & $3.85(1.90)$ \\
\hline Memory score (range $0-8,8$ :completely recalled four items) & $6.18(1.63)$ \\
\hline EQ-5D, baseline [mean (SD)] & $0.82(0.14)$ \\
\hline EQ-5D, 6-month follow-up [mean (SD)] & $0.82(0.15)$ \\
\hline EQ-5D, 12-month follow-up [mean (SD)] & $0.83(0.15)$ \\
\hline
\end{tabular}

Data are expressed as $n(\%)$ unless otherwise specified

PACE Pharmaceutical Assistance Contract for the Elderly, GED General Education Development Certificate, $E Q-5 D$ EuroQoL five-dimension questionnaire, $S D$ standard deviation

${ }^{\mathrm{a}} n=425$

${ }^{\mathrm{b}} n=424$

${ }^{c} n=368$

${ }^{\mathrm{d}}$ Scores were assessed using 5-point Likert scales ranging from 1 (excellent) to 5 (poor)

\subsection{Statistical Analyses}

We hypothesized that higher scores in all four PHM scales would be associated with lower EQ-5D index scores, after adjusting for the baseline covariates. Descriptive statistics and simple linear regression for each explanatory variable were performed to assess data properties and potential associations between each explanatory variable and the EQ-5D index. Considering a longitudinal data structure for the EQ-5D index, generalized mixed-effects models were 
used to investigate EQ-5D index change over time using subject ID as level 2 and measurement time point as level 1. The missing data pattern was analyzed to examine assumptions for mixed effect models. As indicators for model selection, Snijders/Bosker R-squared statistics and the intraclass correlation coefficient (ICC) were used. The residuals of the fitted models were calculated and assessed graphically using histogram and $\mathrm{Q}-\mathrm{Q}$ plots. Multicollinearity was tested based on the variance inflation factor, with a cut-off at 10 . We used a significance level of 0.05 for hypothesis testing. All statistical analyses were performed using STATA/SE 14.2 (StataCorp LLC, College Station, TX, USA).

\section{Results}

A total of 426 community-dwelling adults aged 65 years or older who participated in Falls Free PA at senior centers and had also received at least one prescription through Pennsylvania's PACE program were included in the analysis. Table 1 reveals the participants' baseline demographics and the EQ-5D-3L summary index over time. Of the 17 index conditions, the mean number of comorbidities among study participants was 3.9 (standard deviation [SD] 1.9). The mean EQ-5D-3L summary index at baseline and 6- and 12-month follow-up were 0.82 (SD 0.14), 0.82 (SD 0.15 ), and 0.83 (SD 0.15), respectively.

The mean and distribution of prescription-related variables are summarized in Table 2. The proportion of participants with at least one PHM use was $58.7 \%$ for ACB, $36.6 \%$ for DBI-Se, $24.1 \%$ for DBI-Ach, and $19.2 \%$ for the Beers list. The most common PHMs based on each scale are listed in ESM 5-8. The number of regular medications based on the claims data was 4.1 (SD 3.0, range 0-14), which was concordant with the number of self-reported medications at 6 months' follow-up (see Table 2). Table 3 shows the correlation coefficient between the score of each pair of PHM scales, which ranged from 0.17 (DBI-Se and DBI-Ach) to 0.57 (ACB and DBI-ACh).
After adjusting for covariates, both DBI-Se (coefficients $-0.076,95 \%$ CI -0.131 to -0.020 ) and DBI-Ach (coefficients $-0.095,95 \% \mathrm{CI}-0.188$ to -0.002$)$ significantly predicted a decline in EQ-5D index over time. In addition to the DBI-Se and DBI-Ach, low household income, number of comorbid conditions, the use of assistive devices for walking, and any history of falls in the previous year were all associated with a decline in the EQ$5 \mathrm{D}$ index. The ACB, number of regular medications, and number of Beers medications did not have a significant association with EQ-5D changes during the study period (Table 4). Variable transformations and the use of nonparametric methods did not significantly improve the model fit, and there was no multicollinearity between independent variables included in the fitted models.

\section{Discussion}

Our study indicated a high prevalence of PHM exposure among relatively poor community-dwelling older adults, with variation in prevalence depending on the measure. This was concordant with previous studies that found heterogeneity to be a challenge in advancing research regarding PHMs [31, 32]. In our study, the predictive value of the measures for decline in QoL differed significantly, despite the moderate correlation between scores for each scale.

Head-to-head comparisons of these PHM scales, including polypharmacy, DBI, and ACB, have examined concordance and prediction capacity among the scales. Naples et al. [39] found considerable differences between scales in the estimation of drug burden scores, including the ACB and the DBI. Among the scales that do not consider dose, ACB detected the highest number of medications with anticholinergic effects. Salahudeen et al. compared nine previously published PHM scales and concluded that the DBI-Ach was the strongest predictor of hospital admissions, hospitalizations for falls, and office visits [38]; however, despite previous efforts to identify the

Table 2 Potentially harmful medication-related variables

\begin{tabular}{|c|c|c|c|c|}
\hline PHM variables & Mean & SD & Minimum & Maximum \\
\hline ACB $0-12$ months & 0.55 & 0.87 & 0 & 4.5 \\
\hline DBI-Se $0-12$ months & 0.12 & 0.24 & 0 & 1.51 \\
\hline DBI-Ach $0-12$ months & 0.05 & 0.14 & 0 & 0.94 \\
\hline Number of prescription medications (self-report at 6-month follow-up) & 3.89 & 3.13 & 0 & 15 \\
\hline Number of prescription medications (claims data) ${ }^{\mathrm{a}}$ & 4.13 & 2.99 & 0 & 14 \\
\hline Number of PHMs listed on Beers criteria (claims data) ${ }^{\mathrm{a}}$ & 0.24 & 0.53 & 0 & 3 \\
\hline
\end{tabular}

PHM potentially harmful medication, ACB Anticholinergic Cognitive Burden, DBI-Se Drug Burden Index-sedative property, DBI-Ach Drug Burden Index-anticholinergic property

${ }^{a}$ Number of prescription drugs for which claims were provided for at least 30 days and at least twice during the study period 
Table 3 Correlation matrix between PHM scale scores

\begin{tabular}{llllll}
\hline & ACB & DBI-Se & DBI-ACh & Number of regular medications $^{\mathrm{a}}$ & Number of Beers list medications $^{\mathrm{a}}$ \\
\hline ACB & 1.00 & - & - & - & - \\
DBI-Se & 0.42 & 1.00 & - & - & - \\
DBI-Ach & 0.57 & 0.17 & 1.00 & - & - \\
Number of regular medications $^{\mathrm{a}}$ & 0.55 & 0.42 & 0.32 & 1.00 & - \\
Number of Beers list medications $^{\mathrm{a}}$ & 0.43 & 0.53 & 0.26 & 0.36 & 1.00
\end{tabular}

PHM potentially harmful medication, $A C B$ Anticholinergic Cognitive Burden, DBI-Se Drug Burden Index-sedative property, DBI-Ach Drug Burden Index-anticholinergic property

${ }^{a}$ Number of prescription drugs for which claims were provided for at least 30 days and at least twice during the study period

Table 4 Random intercept model for EQ-5D index over time

\begin{tabular}{|c|c|}
\hline Variables & Final model $[\beta(\mathrm{SE})]$ \\
\hline \multicolumn{2}{|l|}{ Fixed effects } \\
\hline Intercept & $0.993(0.072)^{\dagger \dagger \dagger}$ \\
\hline Age & $-0.001(0.001)$ \\
\hline Sex & $-0.029(0.018)$ \\
\hline Living with someone & $0.001(0.001)$ \\
\hline Income level & $0.033(0.014)^{\dagger \dagger}$ \\
\hline Number of comorbidities & $-0.011(0.003)^{\dagger \dagger}$ \\
\hline Use of assistive devices & $-0.083(0.013)^{\dagger \dagger \dagger}$ \\
\hline Fall in the last year & $-0.035(0.012)^{\dagger \dagger}$ \\
\hline DBI-Se & $-0.076(0.028)^{\dagger \dagger}$ \\
\hline DBI-Ach & $-0.095(0.047)^{\dagger}$ \\
\hline ACB & $0.006(0.009))$ \\
\hline Number of regular medications & $0.001(0.002)$ \\
\hline Number of Beers list medications & $0.016(0.013)$ \\
\hline \multicolumn{2}{|l|}{ Random effects } \\
\hline ID_subjectsd(_cons) & $0.079(0.005)$ \\
\hline Sd (residual) & $0.096(0.003)$ \\
\hline Snijders/Bosker R-squared Level 1 (measurement time point) & 0.218 \\
\hline Snijders/Bosker R-squared Level 2 (subject ID) & 0.316 \\
\hline Intraclass correlation coefficient & 0.405 \\
\hline
\end{tabular}

All variables included in the fitted models are reported in this table

$E Q-5 D$ EuroQoL five-dimension questionnaire, $S E$ standard error, DBI-Se Drug Burden Index-sedative property, DBI-Ach Drug Burden Index-anticholinergic property, $A C B$ Anticholinergic Cognitive Burden ${ }^{\dagger} p<0.05,{ }^{\dagger \dagger} p<0.01,{ }^{\dagger \dagger} p<0.001$ best measurement, there is no consensus regarding the best PHM scale to date.

Similar to the previous literature by Salahudeen et al. [38], our study indicates that DBI-Se and DBI-ACh are stronger predictors for a decline in QoL among community-dwelling older adults compared with ACB, the number of regular medications, and the number of Beers list medications. The fact that ACB, which contains drugs similar to DBI-ACh, did not reveal significant predictive capability may imply that the cumulative drug dose has an important effect on the QoL of older adults. Thus, efforts to reduce overall drug dose, as well as stopping medications, may play a key role in reducing drug-related harms and help avoid unnecessary decline in older adults' health-related QoL.

Although there has been no consensus regarding the minimum clinically important difference of EQ-5D-3L index score [40], Pickard et al. found that an EQ-5D index change of 0.06 was found to be clinically meaningful [41]. According to our final linear random intercept model, a 0.79 -point increase in the cumulative DBI-Se or a 0.63 point increase in the cumulative DBI-ACh was associated with a 0.06 decline in EQ-5D summary index. An older adult taking a DBI-ACh medication on a daily basis at a 
dose twice as high as the minimum effective dose would have a cumulative DBI-ACh increase of 0.66 . Based on these results, it is likely that PHMs in community-dwelling older adults may be responsible for clinically significant declines in health-related QoL.

We also found that the following patient characteristics were associated with a change in QoL over time: patient subjective perception regarding their own economic status, multimorbidity (i.e. the number of baseline chronic conditions), use of an assistive device for walking, and history of previous falls within 1 year. One strength of the current study was that we were able to adjust for these potential confounders. In addition, data gathered from the drug claims database, as well as the linked longitudinal patient cohort follow-up, enabled an accurate and valid estimation of the risk of QoL change.

Our study has several limitations. First, our model assumed complete medication adherence, while no information was available regarding participants' actual medication adherence. Based on the possibility of overestimation of drug adherence, our final model may underestimate the effect of PHM exposure on QoL. Overthe-counter medications and herbal medications were not included in the PHM scales, although current evidence suggests a rise in the use of these medications [5]. In addition, a relatively small sample size might have resulted in non-significant results for ACB, the number of regular medications, and the number of Beers list medications. Furthermore, our results may not be generalizable to a population with higher morbidity due to a potential for prevalent user bias.

\section{Conclusion}

PHM measures incorporating dose revealed a better predictive capacity for QoL change. Reducing cumulative drug dose, as well as stopping harmful/unnecessary medications, would be important for the well-being of this population. These results can be used as the basis for further research and education, with a focus on reducing PHM use in primary care practices, if further validated in different populations.

Acknowledgements This research was supported by Cooperative Agreement Number DP002657 from the Centers for Disease Control and Prevention, Prevention Research Centers program, NIA Grant K01 AG044433, NLM Grant R01 LM011838, and NIH AG024827, Pittsburgh Older Americans Independence Center. The findings and conclusions in this presentation are those of the author and do not necessarily represent the official position of the above-named organizations.

\section{Compliance with Ethical Standards}

Conflicts of interest Kenya Ie, Eric Chou, Richard D. Boyce, Steven M. Albert declare no conflicts of interest.

Open Access This article is distributed under the terms of the Creative Commons Attribution-NonCommercial 4.0 International License (http://creativecommons.org/licenses/by-nc/4.0/), which permits any noncommercial use, distribution, and reproduction in any medium, provided you give appropriate credit to the original author(s) and the source, provide a link to the Creative Commons license, and indicate if changes were made.

\section{References}

1. Charlesworth CJ, Smit E, Lee DSH, Alramadhan F, Odden MC. Polypharmacy among adults aged 65 years and older in the United States: 1988-2010. J Gerontol A Biol Sci Med Sci. 2015;70(8):989-95.

2. Bahat G, Bay I, Tufan A, Tufan F, Kilic C, Karan MA. Prevalence of potentially inappropriate prescribing among older adults: a comparison of the Beers 2012 and Screening Tool of Older Person's Prescriptions criteria version 2. Geriatr Gerontol Int. 2016;17(9):9-12.

3. Narayan SW, Hilmer SN, Horsburgh S, Nishtala PS. Anticholinergic component of the drug burden index and the anticholinergic drug scale as measures of anticholinergic exposure in older people in New Zealand: a population-level study. Drugs Aging. 2013;30(11):927-34.

4. West T, Pruchnicki MC, Porter K, Emptage R. Evaluation of anticholinergic burden of medications in older adults. J Am Pharm Assoc. 2013;53(5):496-504.

5. Jirón M, Pate V, Hanson LC, Lund JL, Jonsson Funk M, Stürmer $\mathrm{T}$. Trends in prevalence and determinants of potentially inappropriate prescribing in the United States: 2007 to 2012. J Am Geriatr Soc. 2016;64(4):788-97.

6. Mintzer J, Burns A. Anticholinergic side-effects of drugs in elderly people. J R Soc Med. 2000;93(9):457-62.

7. Staskin DR, Zoltan E. Anticholinergics and central nervous system effects: are we confused? Rev Urol. 2007;9(4):191-6.

8. Centers for Medicare and Medicaid Services. NHE Fact Sheet. 2015. http://web.archive.org/web/20160325101526/https://www. cms.gov/research-statistics-data-and-systems/statistics-trendsand-reports/nationalhealthexpenddata/nhe-fact-sheet.html. Accessed 18 July 2017.

9. Veehof L, Stewart R, Haaijer-Ruskamp F, Jong BM. The development of polypharmacy. A longitudinal study. Fam Pract. 2000;17(3):261-7.

10. Peterson LE, Bazemore AW, Jr RLP. Family physicians' present and future role. Am Fam Physician. 2009;80(10):1072.

11. Samuel MJ. American Geriatrics Society 2015 updated beers criteria for potentially inappropriate medication use in older adults. J Am Geriatr Soc. 2015;63(11):2227-46.

12. O'Mahony D, O'Sullivan D, Byrne S, O'Connor MN, Ryan C, Gallagher P. STOPP/START criteria for potentially inappropriate prescribing in older people: version 2. Age Ageing. 2015;44(2):213-8.

13. Hilmer SN, Mager DE, Simonsick EM, et al. A drug burden index to define the functional burden of medications in older people. Arch Intern Med. 2007;167:781-7. 
14. Hilmer SN, Mager DE, Simonsick EM, et al. Drug burden index score and functional decline in older people. Am J Med. 2009;122(12):1142-1149-2.

15. Gnjidic D, Cumming RG, Le Couteur DG, et al. Drug Burden Index and physical function in older Australian men. Br J Clin Pharmacol. 2009;68(1):97-105.

16. Cao YJ, Mager DE, Simonsick EM, et al. Physical and cognitive performance and burden of anticholinergics, sedatives, and ACE inhibitors in older women. Clin Pharmacol Ther. 2008;83(3):422-9.

17. Best O, Gnjidic D, Hilmer SN, Naganathan V. McLachlan a J. Investigating polypharmacy and drug burden index in hospitalised older people. Intern Med J. 2013;43(8):912-8.

18. Wilson NM, Hilmer SN, March LM, et al. Associations between drug burden index and falls in older people in residential aged care. J Am Geriatr Soc. 2011;59(5):875-80.

19. Boustani MA, Campbell N, Munger S, Fox C. Impact of anticholinergics on the aging brain: a review and practical application. Aging Health. 2008;4(3):311-20.

20. Fox C, Richardson K, Maidment ID, et al. Anticholinergic medication use and cognitive impairment in the older population: the Medical Research Council cognitive function and ageing study. J Am Geriatr Soc. 2011;59(8):1477-83.

21. Pasina L, Djade CD, Lucca U, et al. Association of anticholinergic burden with cognitive and functional status in a cohort of hospitalized elderly: comparison of the anticholinergic cognitive burden scale and anticholinergic risk scale: Results from the REPOSI study. Drugs Aging. 2013;30(2):103-12.

22. Koyama A, Steinman M, Ensrud K, Hillier TA, Yaffe K. Longterm cognitive and functional effects of potentially inappropriate medications in older women. J Gerontol A Biol Sci Med Sci. 2014;69(4):423-9.

23. Fulton MM, Allen ER. Polypharmacy in the elderly: a literature review. J Am Acad Nurse Pract. 2005;17(4):123-32.

24. Viktil KK, Blix HS, Moger TA, Reikvam A. Polypharmacy as commonly defined is an indicator of limited value in the assessment of drug-related problems. Br J Clin Pharmacol. 2007;63(2):187-95.

25. Alhmoud E, Khalifa S, Bahi AA. Prevalence and predictors of potentially inappropriate medications among home care elderly patients in Qatar. Int J Clin Pharm. 2015;37(5):815-21.

26. Bin Chang C, Lai HY, Yang SY, et al. Patient- and clinic visitrelated factors associated with potentially inappropriate medication use among older home healthcare service recipients. PLoS One. 2014;9(4):1-7.

27. Holmes HM, Luo R, Kuo Y-F, Baillargeon J, Goodwin JS. Association of potentially inappropriate medication use with patient and prescriber characteristics in Medicare Part D. Pharmacoepidemiol Drug Saf. 2013;22(7):728-34.

28. Jyrkkä J, Enlund H, Korhonen MJ, Sulkava R, Hartikainen S. Polypharmacy status as an indicator of mortality in an elderly population. Drugs Aging. 2009;26(12):1039-48.

29. Ziere G, Dieleman JP, Hofman A, Pols HAP, Van Der Cammen TJM, Stricker BHC. Polypharmacy and falls in the middle age and elderly population. Br J Clin Pharmacol. 2006;61(2):218-23.

30. Sehgal V, Bajwa SJ, Sehgal R, et al. Polypharmacy and potentially inappropriate medication use as the precipitating factor in readmissions to the hospital. J Fam Med Prim Care. 2013;2(2):194-9.

31. Bosboom PR, Alfonso H, Almeida OP, Beer C. Use of potentially harmful medications and health-related quality of life among people with dementia living in residential aged care facilities. Dement Geriatr Cogn Dis Extra. 2012;2(1):361-71.

32. Franic DM, Jiang JZ. Potentially inappropriate drug use and health-related quality of life in the elderly. Pharmacotherapy. 2006;26(6):768-78.

33. Albert SM, King J, Boudreau R, Prasad T, Lin CJ, Newman AB. Primary prevention of falls: effectiveness of a statewide program. Am J Public Health. 2014;104(5):77-84.

34. Pennsylvania Department of Aging. Prescriptions. Commonwealth of Pennsylvania. 2017. http://www.aging.pa.gov/agingservices/prescriptions/Pages/default.aspx. Accessed 18 July 2017.

35. Pennsylvania Department of Aging. Pharmaceutical assistance contract for the elderly annual report to the Pennsylvania general assembly January 1-December 31, 2014. 2014. http://www. aging.pa.gov/publications/annual-reports/Documents/2014PACE AnnualReport.pdf. Accessed 18 July 2017.

36. EuroQol Group. EuroQol: a new facility for the measurement of health-related quality of life. Health Policy. 1990;16(3):199-208.

37. Szende A, Janssen BCJ. Self-reported population health: an international perspective based on EQ-5D. Dordrecht: Springer; 2014.

38. Salahudeen MS, Hilmer SN, Nishtala PS. Comparison of anticholinergic risk scales and associations with adverse health outcomes in older people. J Am Geriatr Soc. 2015;63(1):85-90.

39. Naples JG, Marcum ZA, Perera S, Gray SL, Newman AB, Simonsick EM. Concordance between anticholinergic burden scales. J Am Geriatr Soc. 2015;63:2120-4.

40. Coretti S, Ruggeri M, McNamee P. The minimum clinically important difference for EQ-5D index: a critical review. Expert Rev Pharmacoecon Outcomes Res. 2014;14(2):221-33.

41. Pickard AS, Neary MP, Cella D. Estimation of minimally important differences in EQ-5D utility and VAS scores in cancer. Health Qual Life Outcomes. 2007;30:2-9. 